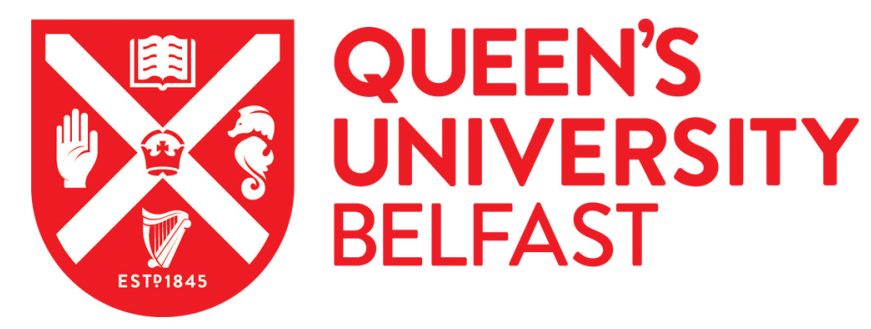

\title{
Visual Field Outcomes from the Multicenter, Randomized Controlled Laser in Glaucoma and Ocular Hypertension Trial (LiGHT)
}

Wright, D., Konstantakopoulou, E., Montesano, G., Nathwani, N., Garg, A., Garway-Heath, D., Crabb, D., \& Gazzard, G. (2020). Visual Field Outcomes from the Multicenter, Randomized Controlled Laser in Glaucoma and Ocular Hypertension Trial (LiGHT). Ophthalmology, 127(10), 1313-1321.

https://doi.org/10.1016/j.ophtha.2020.03.029

Published in:

Ophthalmology

Document Version:

Peer reviewed version

Queen's University Belfast - Research Portal:

Link to publication record in Queen's University Belfast Research Portal

Publisher rights

Copyright 2020 Elsevier.

This manuscript is distributed under a Creative Commons Attribution-NonCommercial-NoDerivs License

(https://creativecommons.org/licenses/by-nc-nd/4.0/), which permits distribution and reproduction for non-commercial purposes, provided the author and source are cited.

\section{General rights}

Copyright for the publications made accessible via the Queen's University Belfast Research Portal is retained by the author(s) and / or other copyright owners and it is a condition of accessing these publications that users recognise and abide by the legal requirements associated with these rights.

Take down policy

The Research Portal is Queen's institutional repository that provides access to Queen's research output. Every effort has been made to ensure that content in the Research Portal does not infringe any person's rights, or applicable UK laws. If you discover content in the Research Portal that you believe breaches copyright or violates any law, please contact openaccess@qub.ac.uk. 


\section{Visual Field Outcomes from LiGHT: Laser in Glaucoma and Ocular}

2 Hypertension, a multicentre, randomised controlled trial.

3

4

Authors: David M. Wrightt ${ }^{1,2}$, Evgenia Konstantakopoulou ${ }^{3,4,5}$, Giovanni Montesano ${ }^{6}$, Neil Nathwani ${ }^{3}$, Anurag Garg ${ }^{3}$, David Garway-Heath ${ }^{3,4}$, David P. Crabb ${ }^{6 *}$, Gus Gazzard ${ }^{3,4}$; on behalf of the LiGHT Trial Study Group.

Affiliations:

1. Centre for Public Health, Queen's University Belfast, Belfast, UK

2. Health Data Research UK

3. NIHR Biomedical Research Centre at Moorfields Eye Hospital NHS Foundation Trust, London, UK

4. Institute of Ophthalmology, University College London, UK

5. Division of Optics and Optometry, University of West Attica, Greece

6. Optometry and Visual Science, School of Health Science, City, University of London, London, UK

* Corresponding author: David.Crabb1@city.ac.uk

Financial support: The LiGHT trial was funded by the National Institute for Health Research Health Technology Assessment Panel (09/104/40) and was sponsored by Moorfields Eye Hospital NHS Foundation Trust. The funding organisation or sponsor had no role in the design or conduct of this research. This report presents independent research commissioned by the NIHR; the views and opinions expressed by the authors in this publication are those of the authors and do not necessarily reflect those of the NHS, the NIHR, MRC, CCF, NETSCC, the HTA or the Department of Health.

DW received funding through an MRC Innovation Fellowship (MR/S003770/1) affiliated with Health Data Research UK, an initiative funded by UK Research and Innovation, Department of Health and Social Care (England) and the devolved administrations, and leading medical research charities.

Running head: Eye drops vs SLT: visual field progression in glaucoma.

\section{Acronyms}

GPA - Guided progression analysis.

IOP - Intra-ocular pressure.

MD - Mean deviation.

OAG - Open angle glaucoma.

$\mathrm{OHT}$ - Ocular hypertension.

OR - Odds Ratio.

PSD - Pattern standard deviation.

PD - Pattern deviation.

SLT - Selective laser trabeculoplasty.

TD - Total deviation.

VF - Visual field. 
37 Publication details

38 PII: S0161-6420(20)30313-4

39 DOI: https://doi.org/10.1016/j.ophtha.2020.03.029

40 Reference: OPHTHA 11182

41 To appear in: Ophthalmology

42 Received Date: 29 October 2019

43 Revised Date: 20 March 2020

44 Accepted Date: 23 March 2020 
47 To compare visual field outcomes of ocular hypertensive and glaucoma patients treated with

48 Medicine- $1^{\text {st }}$ against those treated with selective laser trabeculoplasty (SLT, Laser-1 $1^{\text {st }}$ ).

49

50

51

52

53

54

55

\section{Design}

Secondary analysis of patients from Laser in Glaucoma and Ocular Hypertension (LiGHT), a multicentre randomised controlled trial.

\section{Participants and controls}

344 patients (588 eyes) treated with Medicine- $1^{\text {st }}, 344$ patients (590 eyes) treated with Laser- $1^{\text {st }}$.

\section{Methods}

Visual fields (VFs) were measured using standard automated perimetry and arranged in series (median length and duration: 9 VFs over 48 months). Hierarchical linear models were used to estimate pointwise VF progression rates, which were then averaged to produce a global progression estimate for each eye. Proportions of points and patients in each treatment group with fast $(<-1$ $\mathrm{dB} / \mathrm{y})$ or moderate $(<-0.5 \mathrm{~dB} / \mathrm{y})$ progression were compared using log-binomial regression.

\section{Main outcome measures}

Pointwise and global progression rates of total deviation (TD) and pattern deviation (PD).

\section{Results}

A greater proportion of eyes underwent moderate or fast TD progression in the Medicine- $1^{\text {st }}$ group compared with the Laser- $1^{\text {st }}$ group (26.2\% vs. $16.9 \%$; Risk Ratio, $\left.R R=1.55[1.23,1.93], P<0.001\right)$. A similar pattern was observed for pointwise rates (Medicine- $1^{\text {st }} 26.1 \%$ vs. Laser- $1^{\text {st }} 19.0 \%, R R=1.37$ $[1.33,1.42], P<0.001)$. A greater proportion of pointwise PD rates were categorised as moderate or fast in the Medicine- $1^{\text {st }}$ group (Medicine- $1^{\text {st }} 11.5 \%$ vs. Laser- $1^{\text {st }} 8.3 \%, R R=1.39[1.32,1.46], P<$ 
68 0.001). There was no statistical difference in the proportion of eyes that underwent moderate or

69 fast PD progression (Medicine- $1^{\text {st }} 9.9 \%$ vs. Laser- $1^{\text {st }} 7.1 \%, \mathrm{RR}=1.39[0.95,2.03], P=0.0928$ ).

\section{Conclusion}

71 A slightly larger proportion of ocular hypertensive and glaucoma patients treated with Medicine- $1^{\text {st }}$ underwent rapid VF progression compared with those treated with Laser-1 $1^{\text {st }}$. 


\section{Introduction}

Glaucoma is a progressive optic neuropathy, that left untreated can lead to loss of vision. Glaucoma can have significant implications for patients and is associated with worse vision related quality of life ${ }^{1-4}$. Assessing visual function, typically done by visual field (VF) examination, is vital for clinical management, especially for assessing the effectiveness of treatment in controlling the disease. VF progression will usually drive treatment intensity, as lowering intra-ocular pressure (IOP) is the only currently available treatment to slow the progression of glaucoma ${ }^{5}$.

Thus far, IOP lowering eye drops have been used as a $1^{\text {st }}$-line treatment for glaucoma and ocular hypertension (OHT), but a recent report from the Laser in Glaucoma and Ocular Hypertension (LiGHT) trial showed that selective laser trabeculoplasty (SLT), an outpatient laser procedure for the reduction of IOP, provides better clinical effectiveness and lower treatment intensity among newly diagnosed glaucoma and OHT patients compared to IOP lowering eye drops, and comparable health related quality of life, whilst also being cost-effective ${ }^{6}$.

Although the IOP lowering efficacy of SLT has been extensively compared to that of eye drops $^{7-11}$ and despite a substantial body of research into VF progression in glaucomatous patients, little evidence exists comparing SLT and IOP lowering eye drops in terms of VF outcomes. This study aims to compare VF progression between patients who received SLT to those who received IOP lowering eye drops, as a $1^{\text {st }}$-line treatment for glaucoma and $\mathrm{OHT}$ in the LiGHT trial.

\section{Methods}

\section{Analysis cohort}

Details of the LiGHT trial design and baseline characteristics are described elsewhere ${ }^{12,13}$. Briefly, the LiGHT trial is a multi-centre, randomised controlled trial comparing IOP lowering eye drops to SLT. A total of 718 newly diagnosed, previously untreated $\mathrm{OHT}$ or open angle glaucoma (OAG) patients were randomised to one of two treatment pathways. Patients in the Medicine- $1^{\text {st }}$ group received 
topical IOP lowering eye drops to reduce IOP, whereas patients in the Laser- $1^{\text {st }}$ group received SLT (followed by medication if required as the trial progressed). Subsequent treatment decisions surrounding treatment escalations, repeated SLT or trabeculectomy were conducted according to the study protocol with the aid of a computerised decision algorithm to avoid bias in clinical decision making. The decision support algorithm used in the LiGHT trial has been described in detail previously ${ }^{12,14}$. Patients were treated to eye-specific IOP targets that were determined according to the computer algorithm. Recruitment lasted two years and ended in October 2014. Primary outcomes were reported at three years and additional funding allowed the trial to extend for a further three years.

At each study visit, visual fields (VFs) were measured using the Humphrey Field Analyzer (HFA) with Swedish interactive threshold algorithm standard 24-2 programme (Carl Zeiss Meditec, Dublin, CA, USA). VF measurements were used primarily as an input (along with IOP and optic disc imaging measurements) into decision support software (DSS), which generated eye-specific treatment recommendations at each study visit. The secondary analysis reported here used VFs extracted from the DSS database on $13^{\text {th }}$ December 2018 , as the trial approached the six-year mark. We constructed a longitudinal series of VFs for each study eye and these formed the basis for all analyses. A total of 11,823 VFs were extracted from the database. Of these, we excluded 86 VFs with false positive rates $>14 \%$ as potentially unreliable, and 56 eyes with very short series ( $<5 \mathrm{VFs})$ as these contained little information from which to estimate progression. Following these exclusions there remained 11,563 VFs, approximately equally distributed between treatment groups. A total of 1178 eyes from 688 patients ( $95.8 \%$ of those randomised) were included in this analysis; treatment groups had similar patient baseline characteristics both to each other and to previously reported analyses ${ }^{6,13}$ (Table 1 ). Median follow-up time (Medicine- $1^{\text {st }} 47$ months, Laser- $1^{\text {st }} 49$ months) and VF series length (Medicine- $1^{\text {st }} 5630$ VFs, 9 VFs per eye; Laser- $1^{\text {st }} 5933$ VFs, 10 VFs per eye) were similar across treatment groups. 


\section{Statistical analysis}

We compared VF outcomes between groups by constructing hierarchical linear models describing change in VF measures over time using the visual field data described above. A trend based method of comparison was chosen because it is potentially more sensitive than event based methods such as Guided Progression Analysis (GPA) for detecting progression ${ }^{15,16}$, especially where the number of events is expected to be small as in these early cases. We examined change at each of the 52 measured locations (excluding the blind spot) in each VF series, specifying a random effects structure nesting locations within eyes, within individuals ${ }^{17}$. This accounted for variation in response among locations, due to eye level variation and correlation between eyes within individuals, respectively, whilst pooling information across the entire cohort to produce the most accurate estimates. Fixed effects terms represented baseline values (equivalent to $y$-axis intercept $[\mathrm{dB}]$ ) and rate of change per year (slope; $\mathrm{dB} /$ year) in each treatment group, enabling us to simultaneously evaluate (using the slope by group interaction term) the statistical evidence for a difference in progression rates between groups and to estimate effect size (i.e. difference in slopes) ${ }^{16,18}$.

Two outcome variables were modelled. Total deviation (TD) is the difference of the measured sensitivity at each location from that expected for a patient of that age with no pathology. Pattern deviation (PD) is the TD value at each location adjusted for generalised depression of sensitivity across the VF ${ }^{19}$. Both PD and TD values were extracted from the HFA. Generalised depression and changes in TD may be caused by several non-glaucomatous conditions including cataract, whereas PD is designed to highlight the more localised VF changes found in glaucoma. However, glaucoma almost always has a diffuse component which is ignored by PD, so it is a less sensitive measure than $\mathrm{TD}$ and is prone to underestimation of glaucomatous damage than $\mathrm{TD}^{20}$. Models were fitted in $R$ version 3.5 (R Development Core Team, R Foundation for Statistical Computing, Vienna, Austria).

Alongside pointwise estimates, global estimates of TD and PD progression for each study eye were extracted from the models. For each eye, the estimated rate at each location was extracted; the 
mean of these pointwise rates was calculated to give the global estimate for that eye. Pointwise estimates enable better detection of spatially localised changes, whereas global estimates are useful for describing diffuse changes in sensitivity.

To assess the clinical importance of differences between treatment groups, we categorised estimated progression rates of each location and eye into one of six categories (fast progression: -1 > slope $\mathrm{dB} / \mathrm{y}$, moderate progression: $-1<=$ slope $<-0.5 \mathrm{~dB} / \mathrm{y}$, slow progression: $-0.5<=$ slope $<0 \mathrm{~dB} / \mathrm{y}$, slow improvement: $0<=$ slope $<0.5 \mathrm{~dB} / \mathrm{y}$, moderate improvement: $0.5<=$ slope $<1 \mathrm{~dB} / \mathrm{y}$, fast improvement: slope $>=1 \mathrm{~dB} / \mathrm{y}$. Category boundaries in the progression end (i.e. slope $<0$ ) of the rate distribution were based on those previously reported in studies of glaucoma progression in clinical populations ${ }^{21,22}$. A symmetrical set of boundaries were applied to the improvement end of the distribution as a measure of variability. A tendency towards faster progression and also faster improvement in one treatment group (i.e. a fatter tailed distribution) would indicate greater variability in rates rather than a shift towards faster progression. We used log-binomial (relative risk) regression to compare the proportion of locations and eyes in each group undergoing fast or moderate progression, representing patients at the greatest risk of vision loss. These models were non-hierarchical, with treatment group as the predictor and the outcome being a binary variable indicating whether the estimated rates (from the hierarchical model) were above or below $-0.5 \mathrm{~dB} / \mathrm{y}$. At the other end of the rate distribution, the proportions of locations and eyes undergoing fast or moderate improvement were compared in a similar manner.

We conducted a sensitivity analysis to further investigate the influence of cataract, refitting our models to exclude eyes that underwent cataract removal. Similarly, eyes that underwent trabeculectomy may have experienced a step increase in sensitivity after surgery. We censored VF series for these eyes at time of surgery and refitted the models. local boards at each participating centre. All patients provided written informed consent before 
participation. The study is registered at controlled-trials.com (ISRCTN32038223) and the protocol is available online ${ }^{12}$.

\section{Results}

\section{Total deviation}

176 Estimated mean pointwise total deviation decreased in both the Medicine- $1^{\text {st }}$ and Laser- $1^{\text {st }}$ groups over time (mean and 95\%Cl: Medicine- $1^{\mathrm{st}}=-0.25 \mathrm{~dB} / \mathrm{y}[-0.31,-0.19] ; \mathrm{SLT}=-0.19 \mathrm{~dB} / \mathrm{y}[-0.25,-0.13]$ ). There was little evidence for a difference in mean rates of progression between groups (slope by group interaction term, $t=1.41, P=0.157)$ but the distribution of estimated progression rates did vary by group. Distributions of both pointwise and global estimates were more strongly left skewed in the Medicine- $1^{\text {st }}$ group than in the Laser- $1^{\text {st }}$ group (Figure 1 , global estimates), indicating that greater proportions of locations and eyes in the Medicine- $1^{\text {st }}$ group showed evidence of more rapid progression (Table 2).

One in four eyes underwent moderate or fast progression in the Medicine- $1^{\text {st }}$ group compared with approximately one in six eyes in the Laser- $1^{\text {st }}$ group (Risk Ratio, $R R=1.55[1.23,1.93], P<0.001$ ). Similarly, a greater proportion of locations was categorised as having moderate or fast progression in the Medicine- $1^{\text {st }}$ group $(\mathrm{RR}=1.37[1.33,1.42], P<0.001)$. There was no evidence for a difference between treatment groups in the proportion of eyes that underwent moderate or fast improvement (RR $1.29[0.83,2.04], P=0.266)$. A greater proportion of locations was categorised as having moderate or fast improvement in the Medicine- $1^{\text {st }}$ group $(\mathrm{RR}=1.31[1.24,1.39], P<0.001)$. $P=0.005)$; locations $(\mathrm{RR}=1.25[1.21,1.29], P<0.001)$. Censoring VF series at trabeculectomy had almost no influence on estimated differences between treatment groups (RRs not shown). 


\section{Pattern deviation}

The distribution of progression estimates was similar for pattern deviation but estimated rates were lower and differences between treatment groups were less pronounced than for total deviation. Estimated mean pointwise pattern deviation decreased in both the Medicine- $1^{\text {st }}$ and Laser- $1^{\text {st }}$ groups over time (mean and $95 \% \mathrm{Cl}$ : Medicine- $1^{\text {st }}=-0.12 \mathrm{~dB} / \mathrm{y}[-0.16,-0.09]$; Laser $-1^{\text {st }}=-0.09 \mathrm{~dB} / \mathrm{y}[-0.13,-$ $0.06])$. There was no evidence for a difference in mean rates of progression between groups ( $t=$ $1.19, P=0.236)$ but both pointwise and global estimates were more strongly left skewed in the Medicine- $1^{\text {st }}$ group than in the Laser- $1^{\text {st }}$ group (Figure 2 ).

There was no evidence for a statistical difference between treatment groups in the proportion of eyes that underwent moderate or fast progression (Table 3, RR $=1.39[0.95,2.03], P=0.0928)$. A greater proportion of locations was categorised as having moderate or fast progression in the Medicine- $1^{\text {st }}$ group $($ Table $3, R R=1.39[1.32,1.46], P<0.001)$. There was no evidence for a difference between treatment groups in the proportion of eyes that underwent moderate or fast improvement (RR $1.86[0.75,4.64], P=0.181)$. A greater proportion of locations were categorised as having moderate or fast improvement in the Medicine- $1^{\text {st }}$ group ( $\left.\mathrm{RR}=1.37[1.24,1.51], P<0.001\right)$.

Following exclusion of eyes that underwent cataract removal, the differences between treatment groups were attenuated: eyes that underwent moderate or fast progression $(\mathrm{RR}=1.18[0.78,1.77]$, $P=0.436)$; locations $(\mathrm{RR}=1.29[1.22,1.35], P<0.001)$. Censoring VF series at trabeculectomy had almost no influence on estimated differences between treatment groups (RRs not shown).

\section{Baseline sensitivity, IOP and progression rates}

Eyes that underwent fast progression or improvement had lower average sensitivity at baseline than those with intermediate progression or improvement rates (Figure 3). Similarly, eyes that underwent fast progression or improvement had slightly lower IOP targets set at baseline than those with 
intermediate rates (Figure 4). There was no evidence that the distributions of baseline sensitivity or IOP targets differed between treatment groups (Table 1).

\section{Discussion}

This study reports on the VF progression differences between glaucoma/OHT patients treated with Medicine- $1^{\text {st }}$ and patients treated with Laser- $1^{\text {st }}$ in the LiGHT trial. Using TD values, we estimated that one in four eyes had moderate or fast VF progression in the Medicine- $1^{\text {st }}$ group whereas in the Laser- $-1^{\text {st }}$ group this value was about one in six. The difference between groups was less pronounced, with no statistical evidence for a difference, when using PD values. The proportion of pointwise rates that were moderate or fast was slightly greater in the Medicine- $1^{\text {st }}$ group using both PD and TD. These differences were not reflected at the upper ends of the rate distributions for either eyes or locations, indicating that our findings were not the result of greater variability in one or other treatment group.

The results of this study suggest that treating patients with Laser- $1^{\text {st }}$ may delay VF progression in comparison to Medicine- $1^{\text {st }}$. IOP control with eye drops may rely upon patient concordance with treatment; indeed IOP lowering drops have been reportedly available to patients only $69 \%$ of the time, whilst concordance may range between $76-86 \%$ with even lower figures reported for more complex instillation regimes ${ }^{23-25}$. Although self-reported concordance in the LiGHT trial has been high $^{14}$, the possibility of poor concordance having a significant adverse effect on disease control cannot be ruled out as actual dose monitoring was not carried out. However, patients in clinical trials are reported to have higher rates of concordance than those in routine care ${ }^{26}$. Thus the true magnitude and clinical importance of the slowing of VF progression in the Laser- $1^{\text {st }}$ group may be much greater. SLT has also been proposed to provide better diurnal IOP stability, as a result of a continuous effect on the trabecular meshwork ${ }^{27-30}$. This is in contrast to the episodic (and sometimes erratic) administration of medication that may allow greater diurnal fluctuation in IOP, and in turn 
faster disease progression. Even with exact concordance with instillation regimes, there are likely to be long gaps between doses overnight, during which IOP may rise.

We observed differences in VF progression between treatment groups despite the fact that both groups were treated to similar IOP targets. This indicates that monitoring of IOP reduction alone (usually measured during office hours and so potentially unrepresentative of diurnal pressure variation) may be insufficient to predict functional changes indicative of progression. This suggests that clinical trials of new glaucoma treatments should include both IOP and VF related outcomes. Greater differences were observed for TD, hinting that non-glaucomatous changes may have also contributed towards differences between groups. Changes in TD may be caused by a number of nonglaucomatous conditions, such as cataract. Were there higher rates of cataract in the Medicine- $1^{\text {st }}$ group it could partially explain the tendency towards faster TD progression. During the period covered by this analysis, cataracts were removed from $10.9 \%$ of eyes in the Medicine- $1^{\text {st }}$ group and $7.1 \%$ of eyes in the Laser- $1^{\text {st }}$ group. Assuming that cataracts not yet requiring surgery follow this distribution, generalised depression of sensitivity due to lens opacity have contributed towards the differences in TD rate between the two treatment groups. This is consistent with the higher rates of cataract after topical medical treatment of glaucoma previously reported by landmark glaucoma studies $^{31-34}$ and itself may contribute to a significant clinical advantage of a Laser- $1^{\text {st }}$ compared to a Medicine- $1^{\text {st }}$ protocol. Our sensitivity analysis showed that differences between treatment groups were narrowed when eyes that underwent cataract removals were excluded. PD models were as strongly influenced by the exclusions as TD models. For example, following the exclusions there was no statistical evidence for a difference in the proportion of eyes undergoing fast or moderate PD progression (there remained strong evidence for a difference in the proportion of locations with moderate or fast progression). This may indicate that as well as having lower sensitivity than TD ${ }^{20}$, PD may not be immune to the influence of cataract. Alternatively, the similar responses of TD and PD following exclusions may indicate that cataract was not driving the between group differences. Instead, cataract formation may be associated with faster glaucoma progression (with oxidative 
stress a potential biological basis for the association) and by excluding cataract removal eyes much of the glaucoma signal may have been excluded also. Considering that we still found clinically relevant differences between treatment groups following exclusion of eyes from which cataracts were removed, and recognising the limitations of both TD and PD, we conclude that greater incidence of both cataract-related and glaucomatous progression in the Medicine- $1^{\text {st }}$ group is likely to have contributed towards the observed differences between treatment groups.

To our knowledge this is the first study to robustly compare VF outcomes between IOP lowering drops and SLT, as previous research has focused on IOP lowering alone as a surrogate for disease control. In the absence of a universally accepted, standardised classification of rates of visual field progression we have adopted that used by Chauhan et al. ${ }^{21}$ : fast progressors as $<-1 \mathrm{~dB} /$ year ($1 \mathrm{~dB} /$ year is approximately ten times faster than age related decay). Although statistical methods differ among studies, our estimates of global TD progression are broadly comparable with MD rates in clinical glaucoma populations, which report median progression rates ranging from $-0.62 \mathrm{~dB} /$ year to $-0.05 d B /$ year $)^{21,35,36}$. For the formal comparisons of Medicine- $1^{\text {st }}$ vs. Laser- $1^{\text {st }}$ we reported the proportion of eyes with moderate or fast progression, combining these categories to ensure reasonable data support for each outcome. These figures are not directly comparable with the number of VF progressions reported in the recent paper on the primary outcomes of $\mathrm{LiGHT}^{6}$, where progression was detected using GPA. The proportions reported here are larger, possibly because trend based methods are more sensitive for detecting progression than event based methods such as $\mathrm{GPA}^{15}$, especially given the relatively high upper threshold of the moderate/fast classification ($0.5 \mathrm{~dB} /$ year). Also, this analysis covers a longer follow-up period, extending beyond the 36 -month point reported previously and so a larger proportion of eyes would be expected to show evidence of VF progression in our study. Despite these methodological differences, both analyses report higher risks of VF progression in the Medicine- $1^{\text {st }}$ group, that may be related to the higher rates of disease deterioration previously reported ${ }^{6}$. 
This VF analysis is more detailed than those previously reported for LiGHT $6,14,37$ in that pointwise rates were modelled and then averaged to produce global rate estimates, retaining more information than if global VF measures such as MD or Pattern Standard Deviation (PSD) had been used. Furthermore, we considered the overall shapes of the progression rate distributions rather than using the mean of each distribution as the single point of comparison. We show that differences between treatment groups were manifest only towards the more rapidly progressing end of the rate distribution. If we had concentrated solely on mean TD and PD we would have found no differences between treatment groups, consistent with the MD and PSD results reported at 36months $s^{14}$.

The data derived for this study were drawn from a carefully conducted, randomised controlled trial. Patients were monitored according to routine clinical care; the trial used eye specific IOP targets which were objectively defined and adjusted by a computerised decision algorithm to avoid bias ${ }^{12}$. Similarly, to avoid bias in clinical decision making, treatment escalation decisions were initiated by the computerised decision algorithm, which followed a robust protocol developed according to international guidelines by the EGS, American Academy of Ophthalmology Preferred Practice Pattern and the and the South-East Asia Glaucoma Interest Group ${ }^{38-40}$. The decision support algorithm used in the LiGHT trial has been described in detail before ${ }^{12,14}$. The success of this strategy is highlighted by the well matched distributions of baseline damage and IOP targets between treatment groups (Table 1, Figures 3 and 4). As a result, any differences in VF progression between treatment groups reflect genuine change, in the presence of identical IOP control practices between the two groups. Patients treated with Laser- $1^{\text {st }}$ exhibited slower VF progression, as shown in this study, in addition to better IOP control, less intense medical and surgical treatment and lower rates of disease deterioration ${ }^{6}$. 
cost-savings for the NHS. With slower VF deterioration SLT may delay or completely avert the need

319 for more intense medical and surgical intervention in a significant proportion of patients.

320 Acknowledgements

321 We thank Dr Amanda Davis for supporting trial management, Emily Dowse and Karine Girard-

322 Claudon for clinical support. We also thank the LiGHT participants and all recruiting sites. 
1. Haymes SA, LeBlanc RP, Nicolela MT, Chiasson LA, Chauhan BC. Risk of Falls and Motor Vehicle Collisions in Glaucoma. Invest Ophthalmol Vis Sci. 2007;48(3):1149-1155. doi:10.1167/iovs.060886

2. Haymes SA, LeBlanc RP, Nicolela MT, Chiasson LA, Chauhan BC. Glaucoma and On-Road Driving Performance. Invest Ophthalmol Vis Sci. 2008;49(7):3035-3041. doi:10.1167/iovs.07-1609

3. Bunce $C$, Wormald R. Leading causes of certification for blindness and partial sight in England \& Wales. BMC Public Health. 2006;6(1):58. doi:10.1186/1471-2458-6-58

4. Crabb DP. A view on glaucoma-are we seeing it clearly? Eye. 2016;30(2):304-313. doi:10.1038/eye.2015.244

5. Garway-Heath DF, Crabb DP, Bunce C, et al. Latanoprost for open-angle glaucoma (UKGTS): a randomised, multicentre, placebo-controlled trial. The Lancet. 2015;385(9975):1295-1304. doi:10.1016/S0140-6736(14)62111-5

6. Gazzard G, Konstantakopoulou E, Garway-Heath D, et al. Selective laser trabeculoplasty versus eye drops for first-line treatment of ocular hypertension and glaucoma (LiGHT): a multicentre randomised controlled trial. The Lancet. 2019;0(0). doi:10.1016/S0140-6736(18)32213-X

7. Nagar M, Ogunyomade A, O'Brart DPS, Howes F, Marshall J. A randomised, prospective study comparing selective laser trabeculoplasty with latanoprost for the control of intraocular pressure in ocular hypertension and open angle glaucoma. Br J Ophthalmol. 2005;89(11):14131417. doi:10.1136/bjo.2004.052795

8. Francis BA, lanchulev T, Schofield JK, Minckler DS. Selective Laser Trabeculoplasty as a Replacement for Medical Therapy in Open-Angle Glaucoma. Am J Ophthalmol. 2005;140(3):524525. doi:10.1016/j.ajo.2005.02.047

9. Patel V, El Hawy E, Waisbourd $M$, et al. Long-term outcomes in patients initially responsive to selective laser trabeculoplasty. Int J Ophthalmol. 2015;8(5):960-964. doi:10.3980/j.issn.22223959.2015.05.19

10. Katz L, Steinmann W, Kabir A, Molineaux J, Wizov S, Marcellino G. Selective Laser Trabeculoplasty Versus Medical Therapy as Initial Treatment of Glaucoma: A Prospective, Randomized Trial. J Glaucoma. 2012;21(7):460-468. doi:10.1097/IJG.0b013e318218287f

11. Lai JS, Chua JK, Tham CC, Lam DS. Five-year follow up of selective laser trabeculoplasty in Chinese eyes. Clin Experiment Ophthalmol. 2004;32(4):368-372. doi:10.1111/j.14429071.2004.00839.x

12. Gazzard G, Konstantakopoulou E, Garway-Heath D, et al. Laser in Glaucoma and Ocular Hypertension (LiGHT) trial. A multicentre, randomised controlled trial: design and methodology. Br J Ophthalmol. 2018;102(5):593-598. doi:10.1136/bjophthalmol-2017-310877

13. Konstantakopoulou E, Gazzard G, Vickerstaff V, et al. The Laser in Glaucoma and Ocular Hypertension (LiGHT) trial. A multicentre randomised controlled trial: baseline patient characteristics. Br J Ophthalmol. 2018;102(5):599-603. doi:10.1136/bjophthalmol-2017-310870 
14. Gazzard G, Konstantakopoulou E, Garway-Heath D, et al. Selective laser trabeculoplasty versus drops for newly diagnosed ocular hypertension and glaucoma: the LiGHT RCT. Health Technol Assess. 2019;23(31):1-102. doi:10.3310/hta23310

15. Garway-Heath DF, Zhu H, Cheng Q, et al. Combining Optical Coherence Tomography with Visual Field Data to Rapidly Detect Disease Progression in Glaucoma: A Diagnostic Accuracy Study. NIHR Journals Library; 2018.

16. Wu Z, Crabb DP, Chauhan BC, Crowston JG, Medeiros FA. Improving the Feasibility of Glaucoma Clinical Trials Using Trend-Based Visual Field Progression End Points. Ophthalmol Glaucoma. 2019;2(2):72-77. doi:10.1016/j.ogla.2019.01.004

17. Bryan SR, Eilers PHC, Lesaffre EMEH, Lemij HG, Vermeer KA. Global Visit Effects in Point-Wise Longitudinal Modeling of Glaucomatous Visual Fields. Invest Ophthalmol Vis Sci. 2015;56(8):4283-4289. doi:10.1167/iovs.15-16691

18. Liu X, Kelly SR, Montesano G, et al. Evaluating the Impact of Uveitis on Visual Field Progression Using Large-Scale Real-World Data. Am J Ophthalmol. 2019;207:144-150. doi:10.1016/j.ajo.2019.06.004

19. Sample PA, Dannheim F, Artes PH, et al. Imaging and Perimetry Society Standards and Guidelines. Optom Vis Sci. 2011;88(1):4. doi:10.1097/OPX.0b013e3181fc3735

20. Artes PH, Nicolela MT, LeBlanc RP, Chauhan BC. Visual Field Progression in Glaucoma: Total Versus Pattern Deviation Analyses. Invest Ophthalmol Vis Sci. 2005;46(12):4600-4606. doi:10.1167/iovs.05-0827

21. Chauhan BC, Malik R, Shuba LM, Rafuse PE, Nicolela MT, Artes PH. Rates of Glaucomatous Visual Field Change in a Large Clinical Population. Invest Ophthalmol Vis Sci. 2014;55(7):4135-4143. doi:10.1167/iovs.14-14643

22. Bryan SR, Crabb DP. A New Graphical Tool for Assessing Visual Field Progression in Clinical Populations. Trans/ Vis Sci Technol. 2018;7(1):22. doi:10.1167/tvst.7.1.22

23. Robin AL, Novack GD, Covert DW, Crockett RS, Marcic TS. Adherence in Glaucoma: Objective Measurements of Once-Daily and Adjunctive Medication Use. Am J Ophthalmol. 2007;144(4):533-540.e2. doi:10.1016/j.ajo.2007.06.012

24. Robin AL, Covert D. Does Adjunctive Glaucoma Therapy Affect Adherence to the Initial Primary Therapy? Ophthalmology. 2005;112(5):863-868. doi:10.1016/j.ophtha.2004.12.026

25. Schwartz GF, Quigley HA. Adherence and Persistence with Glaucoma Therapy. Surv Ophthalmol. 2008;53(6 SUPPL.):S57-S68. doi:10.1016/j.survophthal.2008.08.002

26. Henson DB, Shambhu S. Relative Risk of Progressive Glaucomatous Visual Field Loss in Patients Enrolled and Not Enrolled in a Prospective Longitudinal Study. Arch Ophthalmol. 2006;124(10):1405-1408. doi:10.1001/archopht.124.10.1405

27. Prasad N, Murthy S, Dagianis J, Latina M. A Comparison of the Intervisit Intraocular Pressure Fluctuation After 180 and 360 Degrees of Selective Laser Trabeculoplasty (SLT) as a Primary Therapy in Primary Open Angle Glaucoma and Ocular Hypertension. J Glaucoma. 2009;18(2):157-160. doi:10.1097/IJG.0b013e3181752c97 
28. Elsås T, Junk H, Johnsen H. Diurnal Intraocular Pressure After Successful Primary Laser Trabeculoplasty. Am J Ophthalmol. 1991;112(1):67-69. doi:10.1016/S0002-9394(14)76215-4

29. Greenidge KC, Spaeth GL, Fiol-Silva Z. Effect of Argon Laser Trabeculoplasty on the Glaucomatous Diurnal Curve. Ophthalmology. 1983;90(7):800-804. doi:10.1016/S01616420(83)34479-1

30. Tojo N, Oka M, Miyakoshi A, Ozaki H, Hayashi A. Comparison of Fluctuations of Intraocular Pressure Before and After Selective Laser Trabeculoplasty in Normal-tension Glaucoma Patients. J Glaucoma. 2014;23(8). doi:10.1097/IJG.0000000000000026

31. Leske MC, Heijl A, Hussein M, Bengtsson B, Hyman L, Komaroff E. Factors for Glaucoma Progression and the Effect of Treatment: The Early Manifest Glaucoma Trial. Arch Ophthalmol. 2003;121(1):48-56. doi:10.1001/archopht.121.1.48

32. Heijl A, Leske MC, Bengtsson B, Hyman L, Bengtsson B, Hussein M. Reduction of Intraocular Pressure and Glaucoma Progression: Results From the Early Manifest Glaucoma Trial. Arch Ophthalmol. 2002;120(10):1268-1279. doi:10.1001/archopht.120.10.1268

33. Wu S-Y, Nemesure B, Hennis A, Schachat AP, Hyman L, Leske MC. Open-angle Glaucoma and Mortality: The Barbados Eye Studies. Arch Ophthalmol. 2008;126(3):365-370. doi:10.1001/archophthalmol.2007.77

34. Stewart WC, Stewart JA, Nassar QJ, Mychaskiw MA. Cost-effectiveness of Treating Ocular Hypertension. Ophthalmology. 2008;115(1):94-98. doi:10.1016/j.ophtha.2007.01.040

35. Heijl A, Buchholz P, Norrgren G, Bengtsson B. Rates of visual field progression in clinical glaucoma care. Acta Ophthalmol (Copenh). 2013;91(5):406-412. doi:10.1111/j.17553768.2012.02492.x

36. Moraes CGVD, Juthani VJ, Liebmann JM, et al. Risk Factors for Visual Field Progression in Treated Glaucoma. Arch Ophthalmol. 2011;129(5):562-568. doi:10.1001/archophthalmol.2011.72

37. Garg A, Vickerstaff V, Nathwani N, et al. Primary Selective Laser Trabeculoplasty for Open-Angle Glaucoma and Ocular Hypertension: Clinical Outcomes, Predictors of Success, and Safety from the Laser in Glaucoma and Ocular Hypertension Trial. Ophthalmology. 2019;126(9):1238-1248. doi:10.1016/j.ophtha.2019.04.012

38. European Glaucoma Society. Terminology and Guidelines for Glaucoma 3rd Ed. 2008. http://www.eugs.org/eng/EGS_guidelines.asp. Accessed May 12, 2015.

39. SEAGIG. South East Asia Glaucoma Interest Group. Asia Pacific Glaucoma Guidelines. 2003.

40. American Academy of Ophthalmology. Primary Open-Angle Glaucoma: Preferred Practice Pattern. 2005. 


\section{Tables}

435 Table 1. Distribution of cohort characteristics by treatment group. Values given are frequencies

436 unless otherwise marked.

\begin{tabular}{lll} 
& Medicine-1st & Laser-1st \\
\hline Patients & 344 & 344 \\
Male & $180(52.3 \%)$ & $193(56.1 \%)$ \\
Female & $164(47.7 \%)$ & $151(43.9 \%)$ \\
Age in years, mean (SD) & $62.9(11.6)$ & $63.4(12.0)$ \\
OAG & $271(78.8 \%)$ & $266(77.3 \%)$ \\
OHT & $73(21.2 \%)$ & $78(22.7 \%)$ \\
Eyes & 588 & 590 \\
Bilateral cases & $245(71.2 \%)$ & $249(72.4 \%)$ \\
Follow up duration in months, median (IQR) & $47(39,54)$ & $49(42,56)$ \\
Visual fields & 5630 & 5933 \\
Visual fields per eye, median (IQR) & $9(8,11)$ & $10(8,12)$ \\
Interval between fields in days, median (IQR) & $135(83,189)$ & $140(94,189)$ \\
Visual field mean deviation at baseline in dB, median (IQR) & $-2.0(-4.5,-0.5)$ & $-2.2(-4.4,-0.6)$ \\
IOP target at baseline in mmHg, median (IQR) & $18(16,21)$ & $18(16,21)$ \\
Number of cataract removals performed & 64 & 42 \\
\hline
\end{tabular}

437 
Table 2. Distribution of estimated total deviation progression rates by treatment group.

\begin{tabular}{lllll} 
& Locations & & \multicolumn{2}{l}{ Eyes } \\
Progression rate & Medicine-1st & Laser-1st & Medicine-1st Laser-1st \\
\hline Fast $(-1>$ slope dB/y) & $10.2 \%(3115)$ & $6.0 \%(1848)$ & $9.5 \%(56)$ & $5.4 \%(32)$ \\
Moderate $(-1<=$ slope $<-0.5 \mathrm{~dB} / \mathrm{y})$ & $15.9 \%(4864)$ & $13.0 \%(3980)$ & $16.7 \%(98)$ & $11.5 \%(68)$ \\
Slow $(-0.5<=$ slope $<0 \mathrm{~dB} / \mathrm{y})$ & $40.3 \%(12336)$ & $43.4 \%(13311)$ & $41.5 \%(244)$ & $48.1 \%(284)$ \\
$\begin{array}{l}\text { Slow improvement } \\
(0<=\text { slope }<0.5 \mathrm{~dB} / \mathrm{y})\end{array}$ & $25.7 \%(7863)$ & $31.6 \%(9705)$ & $25.5 \%(150)$ & $29.7 \%(175)$ \\
$\begin{array}{l}\text { Moderate improvement } \\
(0.5<=\text { slope }<1 \mathrm{~dB} / \mathrm{y})\end{array}$ & $5.9 \%(1798)$ & $4.7 \%(1442)$ & $5.1 \%(30)$ & $4.1 \%(24)$ \\
Fast improvement $($ slope $>=1 \mathrm{~dB} / \mathrm{y})$ & $2.0 \%(600)$ & $1.3 \%(394)$ & $1.7 \%(10)$ & $1.2 \%(7)$ \\
\hline
\end{tabular}




\begin{tabular}{|c|c|c|c|c|}
\hline \multirow[b]{2}{*}{ Progression rate } & \multicolumn{2}{|l|}{ Locations } & \multicolumn{2}{|l|}{ Eyes } \\
\hline & Medicine-1st & Laser-1st & Medicine-1st & Laser-1st \\
\hline Fast $(-1>$ slope $\mathrm{dB} / \mathrm{y})$ & $4.6 \%(1403)$ & $3.2 \%(967)$ & $3.4 \%(20)$ & $1.7 \%(10)$ \\
\hline Moderate $(-1<=$ slope $<-0.5 \mathrm{~dB} / \mathrm{y})$ & $6.9 \%(2103)$ & $5.1 \%(1565)$ & $6.5 \%(38)$ & $5.4 \%(32)$ \\
\hline Slow $(-0.5<=$ slope $<0 \mathrm{~dB} / \mathrm{y})$ & $46.6 \%(14234)$ & $48.9 \%(14990)$ & $51.7 \%(304)$ & $55.6 \%(328)$ \\
\hline $\begin{array}{l}\text { Slow improvement } \\
(0<=\text { slope }<0.5 \mathrm{~dB} / \mathrm{y})\end{array}$ & $38.9 \%(11900)$ & $40.6 \%(12471)$ & $36.2 \%(213)$ & $36.1 \%(213)$ \\
\hline $\begin{array}{l}\text { Moderate improvement } \\
(0.5<=\text { slope }<1 \mathrm{~dB} / \mathrm{y})\end{array}$ & $2.6 \%(805)$ & $1.8 \%(557)$ & $2.2 \%(13)$ & $1.0 \%(6)$ \\
\hline Fast improvement (slope $>=1 \mathrm{~dB} / \mathrm{y}$ ) & $0.4 \%(131)$ & $0.4 \%(130)$ & $-(0)$ & $0.2 \%(1)$ \\
\hline
\end{tabular}


$442 \quad$ Figures

443 Figure 1. Distribution of estimated global total deviation progression rates by treatment group.

444 Histogram with median and $10^{\text {th }}$ percentiles indicated. Curved line represents a smoothed density

445 estimate to the histogram.

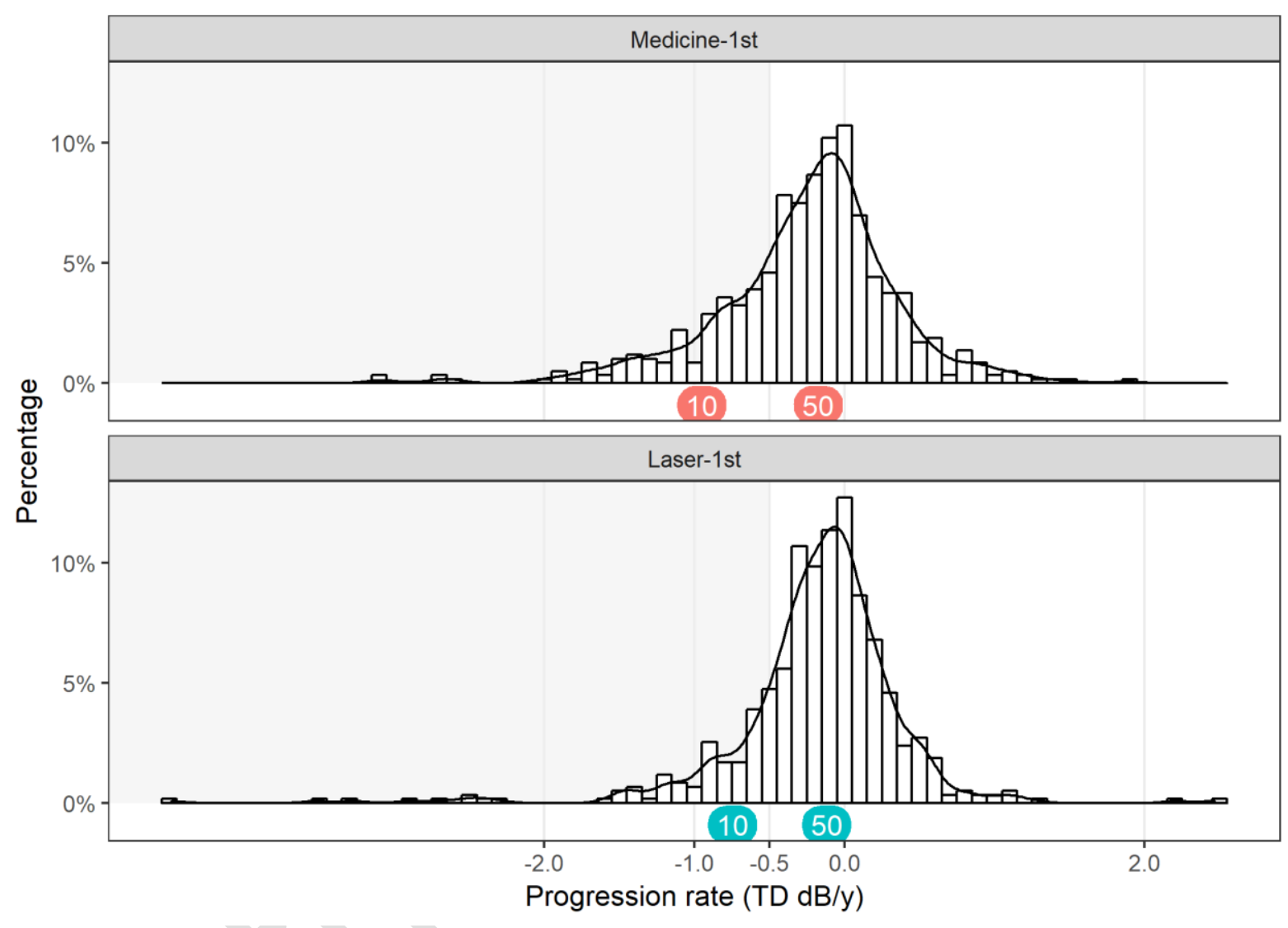


Figure 2. Distribution of estimated global pattern deviation progression rates by treatment group. Histogram with median and $10^{\text {th }}$ percentiles indicated. Curved line represents a smoothed density estimate to the histogram.

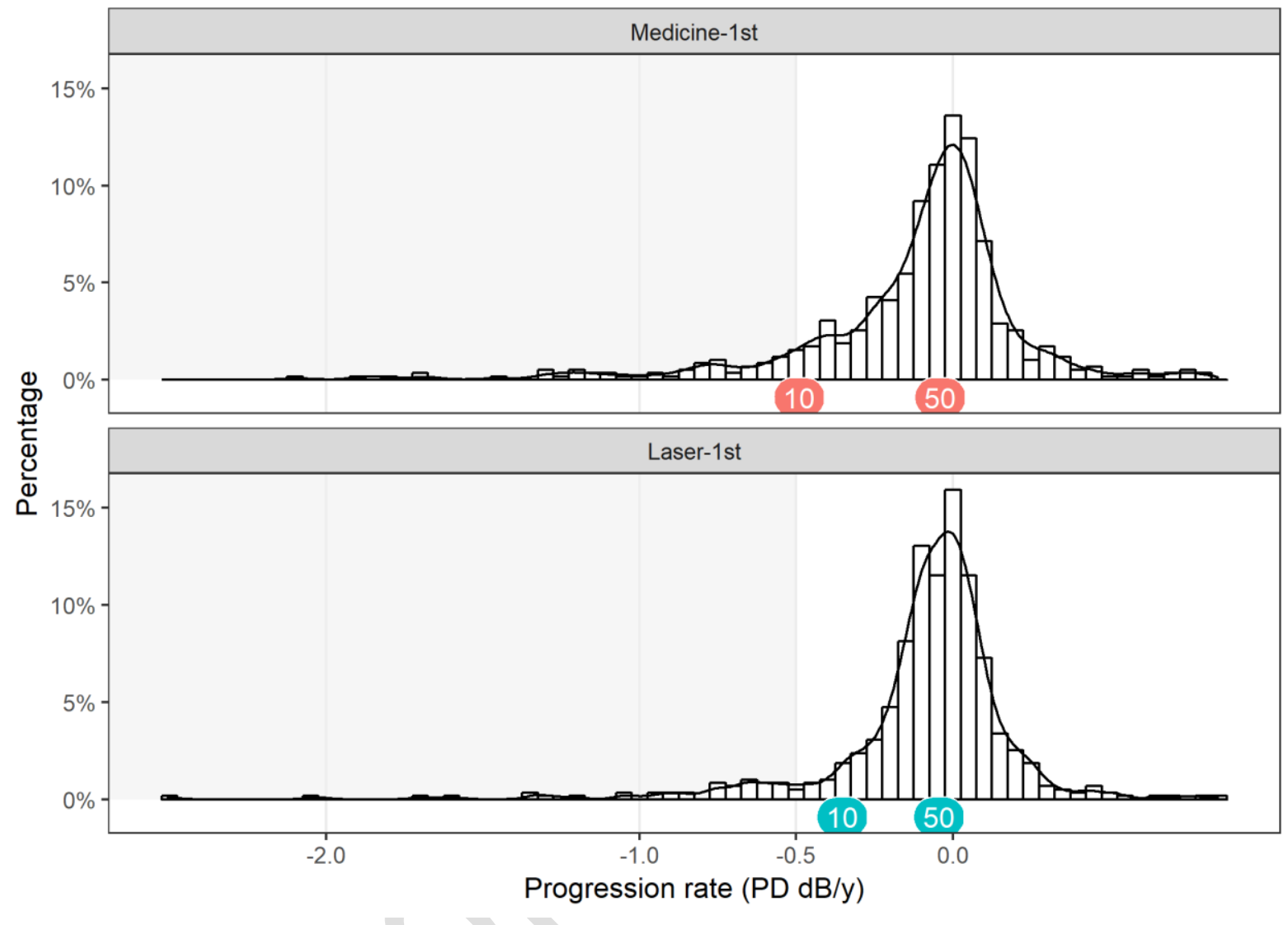

451 

rates.

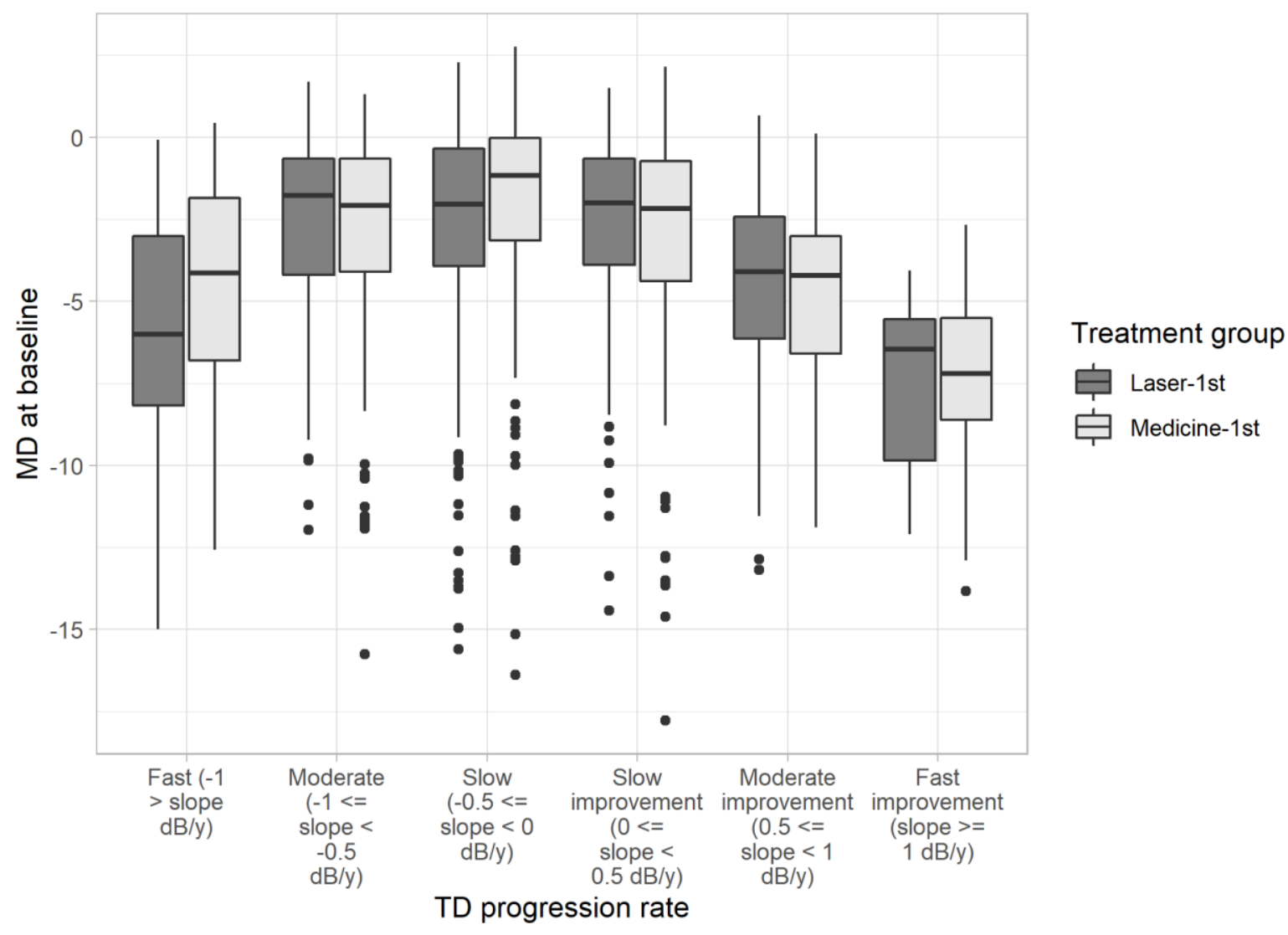


Figure 4. Distribution of target IOP at baseline by estimated total deviation progression rates.

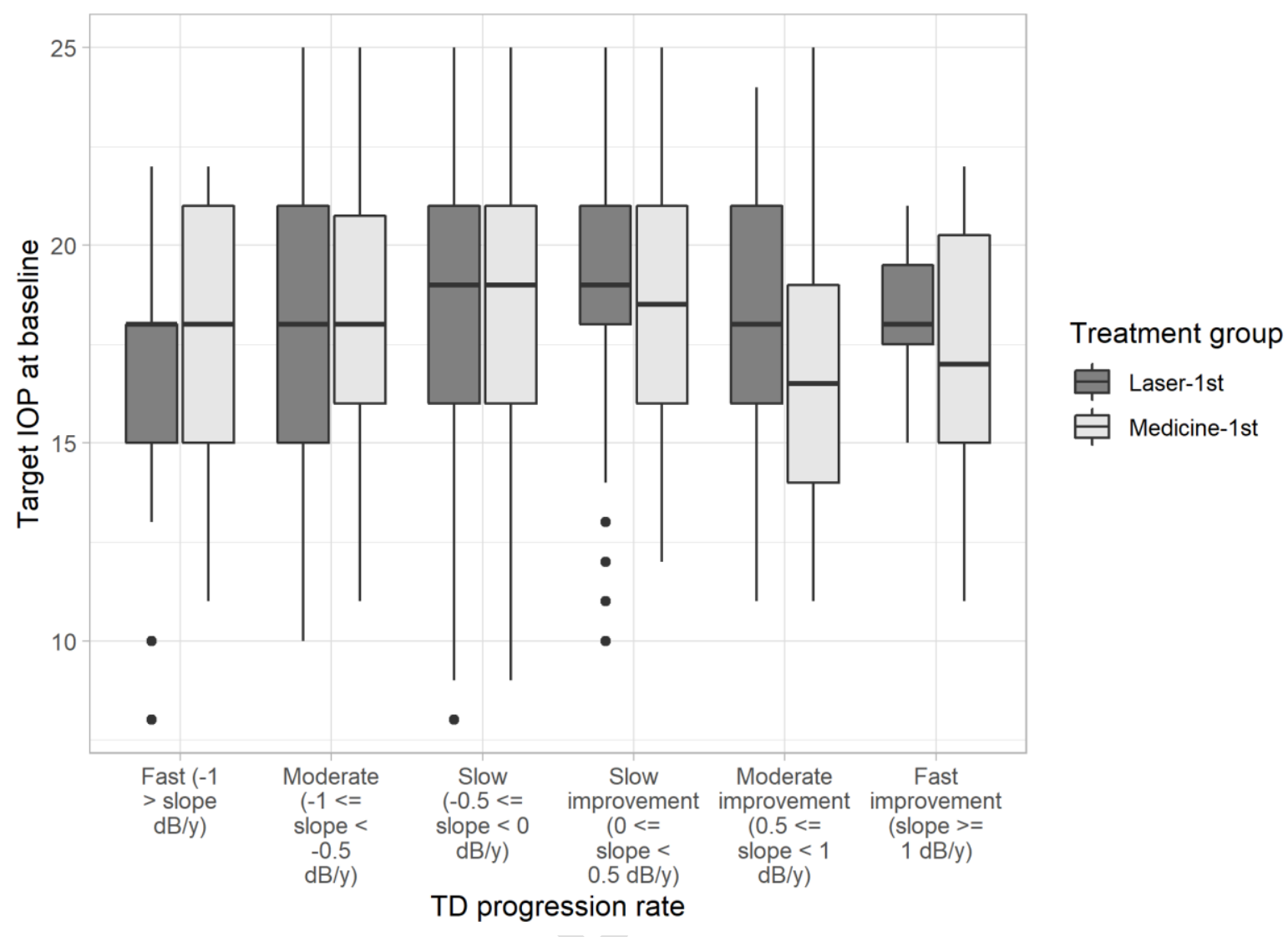

